\title{
Subbrow Approach as a Minimally Invasive Reduction Technique in the Management of Frontal Sinus Fractures
}

\author{
Yewon Lee ${ }^{1}$, Hyun Gon $\mathrm{Choi}^{1}$, Dong Hyeok Shin ${ }^{1}$, Ki Il Uhm ${ }^{1}$, Soon Heum Kim², \\ Cheol Keun $\mathrm{Kim}^{2}$, Dong In Jo ${ }^{2}$ \\ ${ }^{I}$ Department of Plastic and Reconstructive Surgery, Konkuk University Medical Center, Konkuk University School of Medicine, Seoul; \\ ${ }^{2}$ Department of Plastic and Reconstructive Surgery, Konkuk University School of Medicine, Chungju, Korea
}

Background Frontal sinus fractures, particularly anterior sinus fractures, are relatively common facial fractures. Many agree on the general principles of frontal fracture management; however, the optimal methods of reduction are still controversial. In this article, we suggest a simple reduction method using a subbrow incision as a treatment for isolated anterior sinus fractures.

Methods Between March 2011 and March 2014, 13 patients with isolated frontal sinus fractures were treated by open reduction and internal fixation through a subbrow incision. The subbrow incision line was designed to be precisely at the lower margin of the brow in order to obtain an inconspicuous scar. A periosteal incision was made at $3 \mathrm{~mm}$ above the superior orbital rim. The fracture site of the frontal bone was reduced, and bone fixation was performed using an absorbable plate and screws.

Results Contour deformities were completely restored in all patients, and all patients were satisfied with the results. Scars were barely visible in the long-term follow-up. No complications related to the procedure, such as infection, uncontrolled sinus bleeding, hematoma, paresthesia, mucocele, or posterior wall and brain injury were observed.

Conclusions The subbrow approach allowed for an accurate reduction and internal fixation of the fractures in the anterior table of the frontal sinus by providing a direct visualization of the fracture. Considering the surgical success of the reduction and the rigid fixation, patient satisfaction, and aesthetic problems, this transcutaneous approach through a subbrow incision is concluded to be superior to the other reduction techniques used in the case of an anterior table frontal sinus fracture.

Keywords Surgical procedures, minimally invasive / Fracture fixation, internal / Frontal sinus
Correspondence: Hyun Gon Choi Department of Plastic and Reconstructive Surgery, Konkuk University School of Medicine, 120-1 Neungdong-ro, Gwangjin-gu, Seoul 143-729, Korea

Tel: $+82-2-2030-5235$

Fax: +82-2-2030-5249

E-mail:20040059@kuh.ac.kr

No potential conflict of interest relevant to this article was reported.

\section{INTRODUCTION}

Frontal bone fractures account for $5 \%$ to $15 \%$ of all facial frac- tures, with motorcycle accidents being the most common mechanism of injury [1-3]. Frontal fractures are grouped into three distinct general categories: anterior table fractures, posterior ta-

Copyright $\odot 2014$ The Korean Society of Plastic and Reconstructive Surgeons

This is an Open Access article distributed under the terms of the Creative Commons Attribution Non-Commercial License (http://creativecommons.org/

licenses/by-nc/3.0/) which permits unrestricted non-commercial use, distribution, and reproduction in any medium, provided the original work is properly cited.

www.e-aps.org 
ble fractures, and combined fractures $[3,4]$. Among these, isolated anterior table fractures account for $33 \%$ to $39 \%$ of frontal sinus fractures [5].

Many agree on the general principles of frontal fracture management as described below, but the methods of reduction are still controversial [1-9]. In cases of non-displaced anterior or posterior wall fractures, seven days of antibiotics without surgical intervention is recommended. A follow-up computed tomography (CT) scan should be performed in order to confirm any complications or sequelae. During follow-up, cerebrospinal fluid (CSF) leakage may persist due to a posterior wall fracture, which necessitates craniotomy or dural repair procedures. Displaced posterior wall fractures require a reduction of the fracture with additional treatments. In case of concomitant CSF leakage or frontonasal duct injury, the reduction and fixation of the fracture with dural repair and obliteration of the sinus and the frontonasal duct should be performed, and cranialization may be considered. Such complicated procedures are best performed through a coronal incision $[8,9]$. If there are only displaced anterior table fractures with an intact frontonasal duct, reduction with or without fixation is the treatment of choice. Because an isolated anterior table fracture is the most common fracture type, extensive clinical experience suggests the ideal method for approaching the anterior table [10-14]. However, the management of frontal sinus fractures remains somewhat controversial because finding a balance between an acceptable cosmetic outcome with a minimum scar and rigid fixation via sufficient exposure is not easy.

Here, we describe a simple and effective method using a cosmetic subbrow incision to treat anterior table fractures.

\section{METHODS}

All patients with frontal sinus fractures admitted to the plastic surgery department from March 2011 to March 2014 were treated by a single surgeon using our surgical technique. Medical records were reviewed for the collection of each patient's demographic information, injury characteristics, operative details, and clinical outcomes. Patients with anterior wall fractures were included in this study. Patients were excluded if they had concomitant posterior table fractures, comminuted fractures, or severe fractures that indicate open reduction through a bicoronal incision. Patients with large open wounds on the frontal sinus area were also excluded. The group included 12 males and one female, with an average age of 24.3 years (range, 18-54 years). The patients were initially evaluated by neurosurgeons and subsequently assessed by plastic surgeons in order to determine whether open reduction was appropriate. The follow-up dura- tion ranged from two months to 16 months.

\section{Technique}

All patients were placed in the supine position under general anesthesia. The incision line was designed following the lower edge of the eyebrow starting slightly medial to the medial limbus axis line to approximately $1.0 \mathrm{~cm}$ medial to the tail of the brow. Supratrochlear and supraorbital nerves and vessels were marked at $1.5 \mathrm{~cm}$ and $2.5 \mathrm{~cm}$ from the midline, preoperatively. In order to obtain an inconspicuous scar, keeping this upper incision precisely at the lower margin of the brow is very important. The incision was made from the skin to the loose areolar tissue using a no. 15 blade, and the dissection was performed between the loose areolar tissue and the periosteum by using an elevator. The superior orbital rim was exposed by gently dissecting the orbital septum and the orbicularis oculi muscle. A periosteal incision was made at $3 \mathrm{~mm}$ from the superior orbital rim. Meticulous dissection was performed around previously marked areas for the identification and preservation of the supraorbital and supratrochlear nerves and vessels. The fracture was visualized by dissecting the periosteum using an elevator (Fig. 1). The fracture site of the frontal bone was reduced using a periosteal elevator and bone hook. The intact nasofrontal duct was confirmed by probing. The exposed sinus mucosa was then excised conservatively. After the reduction of the fractured segment was accomplished, bone fixation was performed using an absorbable plate and screws. We skipped screwing near the nerve pedicles and only screwed on the surrounding bone fragments in order to

Fig. 1. Fracture site exposure through a subbrow incision

Preoperative marking at $1.5 \mathrm{~cm}$ and $2.5 \mathrm{~cm}$ from the midline $(\mathrm{ML})$ to avoid an injury of the supratrochlear and supraorbital nerves (STN, SON) and vessels. STN and SON are identified and preserved at the marked areas.

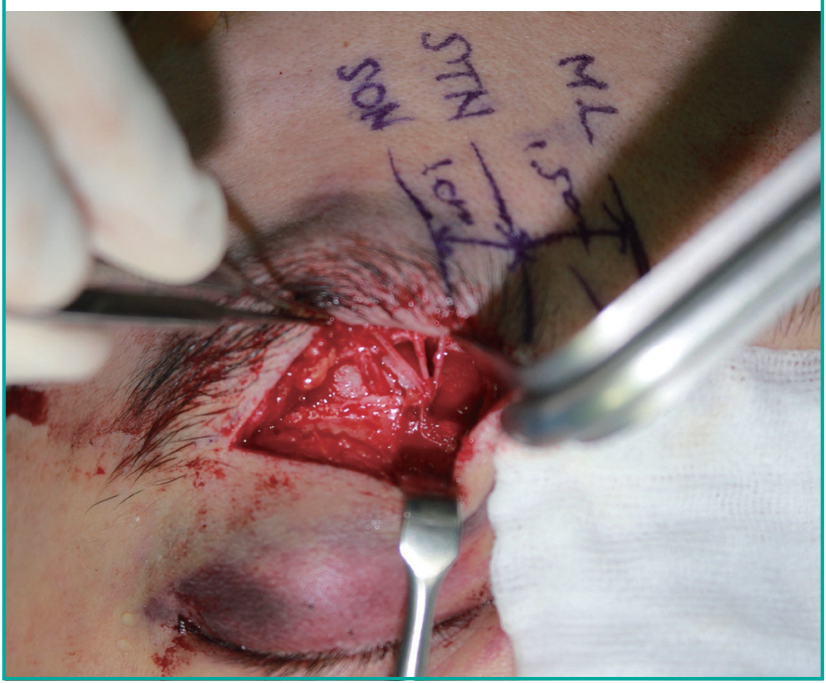


minimize the nerve injury during fixation (Fig. 2). When the fractures were located in the center of the forehead, screw fixation through the incision was very difficult. In these cases, a plate was placed at the fracture site through the subbrow incision and an additional 5-mm midline glabella incision was made only for the screw fixation. Monosyn \#5-0 was used for the repair of the periosteum and the musculoaponeurotic layers. The skin was repaired with interrupted and continuous sutures. Skin sutures were removed on the fifth postoperative day.

\section{RESULTS}

Displaced bone segments were reduced and fixated well in all patients, and postoperative CT scans showed no evidence of the

\section{Fig. 2. Fracture reduction and fixation}

Internal fixation is performed through a subbrow incision using an absorbable plate and screws.

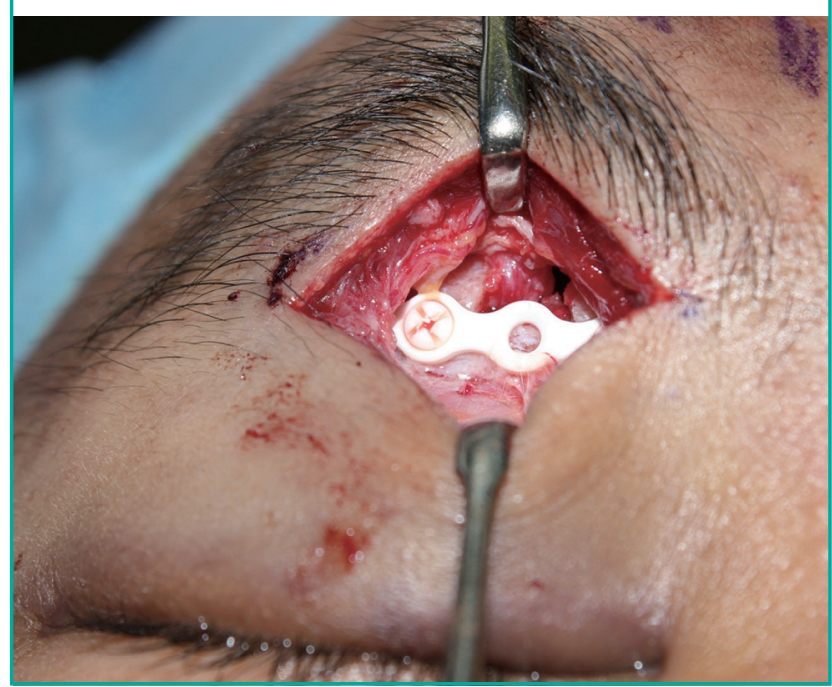

recurring displacement. Access to frontal sinus fracture lines through the subbrow incision was possible in all patients. However, three out of 13 patients needed an additional slit incision at the ipsilateral glabella for screw fixation. All patients showed complete restoration of the contour deformities and were satisfied with the results. Scars were barely visible in the long-term follow-up. No complications related to the procedure, such as infection, uncontrolled sinus bleeding, hematoma, paresthesia, mucocele, or posterior wall and brain injury were observed. Seven patients presented paresthesia in the supratrochlear or supraorbital nerve distribution preoperatively due to nerve compression by the fractured fragments. It subsided gradually and disappeared approximately two months after the operation. The other six patients did not develop any numbness or abnormal sensation in the forehead postoperatively. The follow-up duration ranged from two to 16 months with an average of five months (Table 1).

\section{Case 1}

A 30-year-old man sustained a high-speed traffic accident injury (Fig. 3). The CT scan showed a depressed fracture of the anterior table of the left frontal sinus and nasal bone. There was no evidence of a nasofrontal duct or posterior table injury. A 4-cm subbrow incision was made in order to approach the frontal sinus. A $4 \mathrm{~cm} \times 3 \mathrm{~cm}$ depressed fracture was pried up by a periosteal elevator and raised with a bone hook through the fractured site. Internal fixation was performed using a three-hole absorbable plate and two absorbable screws. We omitted one screw fixation near the nerve pedicle to avoid nerve injury. The postoperative CT scan and three-dimensional reconstruction images showed an excellent contour of the forehead. The patient reported numbness in the forehead from the first physical exami-

Table 1. Patient data

\begin{tabular}{|c|c|c|c|c|c|c|c|}
\hline Patient no. & Sex & Age (yr) & Associated injury & Mechanism & $\begin{array}{l}\text { Hospital } \\
\text { day }\end{array}$ & $\begin{array}{l}\text { Follow-up } \\
\text { (mo) }\end{array}$ & $\begin{array}{l}\text { Operation time } \\
\text { (min) }\end{array}$ \\
\hline 1 & Male & 27 & - & Slip and fall & 7 & 4 & 140 \\
\hline 2 & Male & 21 & $\begin{array}{l}\text { Blowout fracture, nasal } \\
\text { bone fracture }\end{array}$ & Assault & 12 & 3 & 50 \\
\hline 3 & Male & 23 & - & Baseball & 4 & 3 & 75 \\
\hline 4 & Male & 25 & - & Assault & 6 & 9 & 70 \\
\hline 5 & Male & 18 & - & Assault & 7 & 4 & 59 \\
\hline 6 & Male & 40 & - & Assault & 12 & 3 & 70 \\
\hline 7 & Male & 21 & Nasal bone fracture & Slip and fall & 7 & 4 & 105 \\
\hline 8 & Male & 19 & - & Assault & 9 & 3 & 148 \\
\hline 9 & Female & 54 & - & Dumbbell & 11 & 4 & 55 \\
\hline 10 & Male & 53 & - & Slip and fall & 6 & 6 & 115 \\
\hline 11 & Male & 49 & Nasal bone fracture & Traffic accident & 16 & 4 & 122 \\
\hline 12 & Male & 30 & - & Traffic accident & 8 & 16 & 84 \\
\hline 13 & Male & 29 & - & Baseball & 7 & 3 & 128 \\
\hline
\end{tabular}




\section{Fig. 3. Case 1}

(A) Preoperative photograph shows the slight depression of the left forehead. (B) Preoperative computed tomographic scan shows a depressed fracture of the left anterior table of the frontal sinus. (C) Postoperative photograph at six months shows an excellent contour of the forehead with almost no visible scars. (D) Follow-up computed tomographic scan showed adequate reduction.
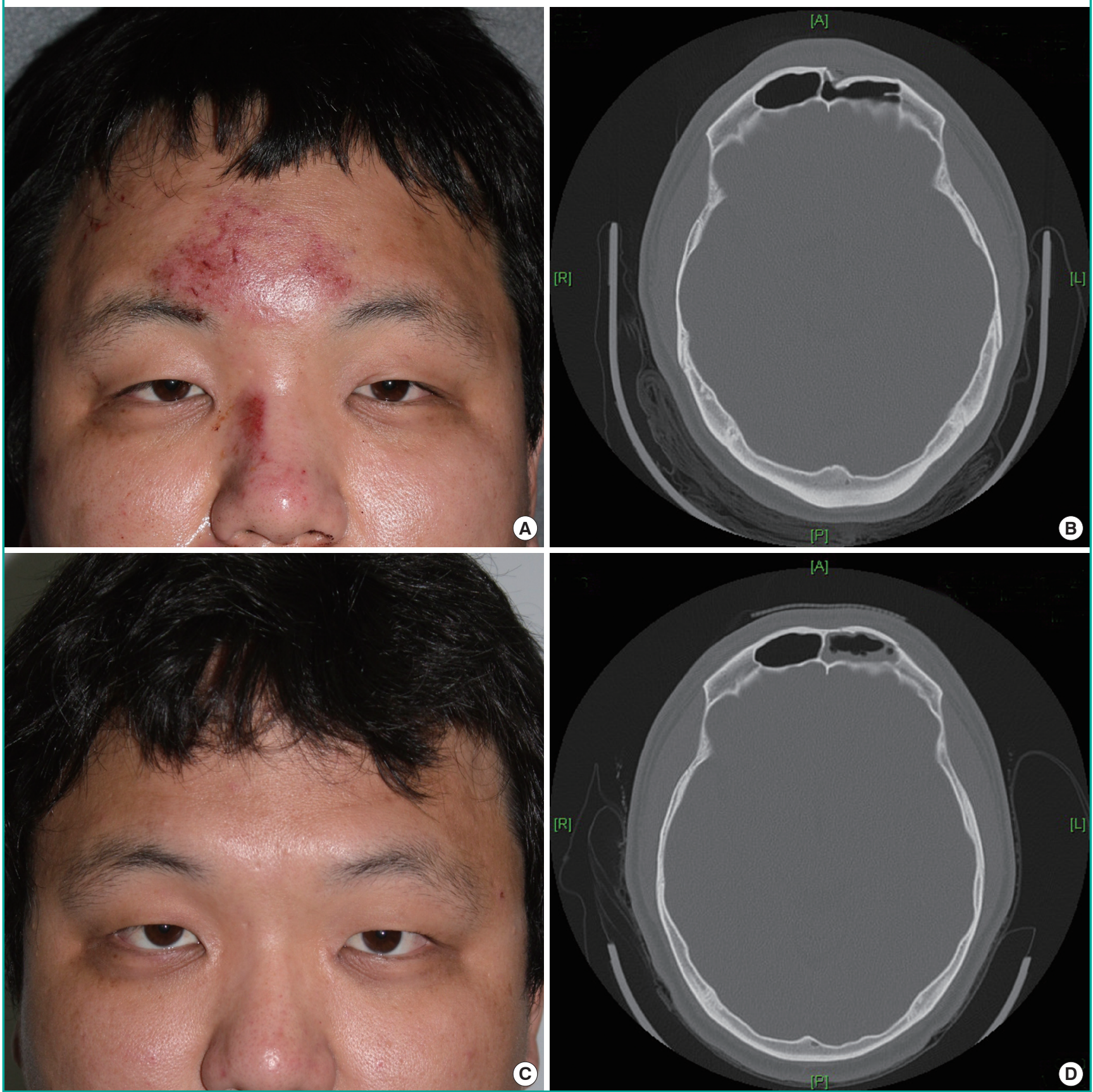

nation preoperatively, but the numbness gradually disappeared by one month after the operation. A postoperative photograph taken on the 16-month follow-up showed that the scars had become almost completely invisible (Fig. 3).

\section{Case 2}

An 18-year-old man suffered a right frontal sinus injury due to physical assault (Fig. 4). There was no open wound. A CT scan showed a depressed fracture of the isolated anterior wall without a nasofrontal duct and posterior table injury. The patient underwent surgery three days after the injury. A 4-cm subbrow incision was made. A $1.5 \mathrm{~cm} \times 1.5 \mathrm{~cm}$ depressed fracture was pried up by a periosteal elevator and raised with a bone hook through the fractured site. Internal fixation was performed using 


\section{Fig. 4. Case 2}

(A) Preoperative photograph shows a slight depression of the right forehead. (B) Preoperative computed tomographic scan shows a depressed fracture of the right anterior table of the frontal sinus. (C) Postoperative photograph at four months shows the restoration of the forehead contour with an inconspicuous scar. (D) Follow-up computed tomographic scan showed adequate reduction.
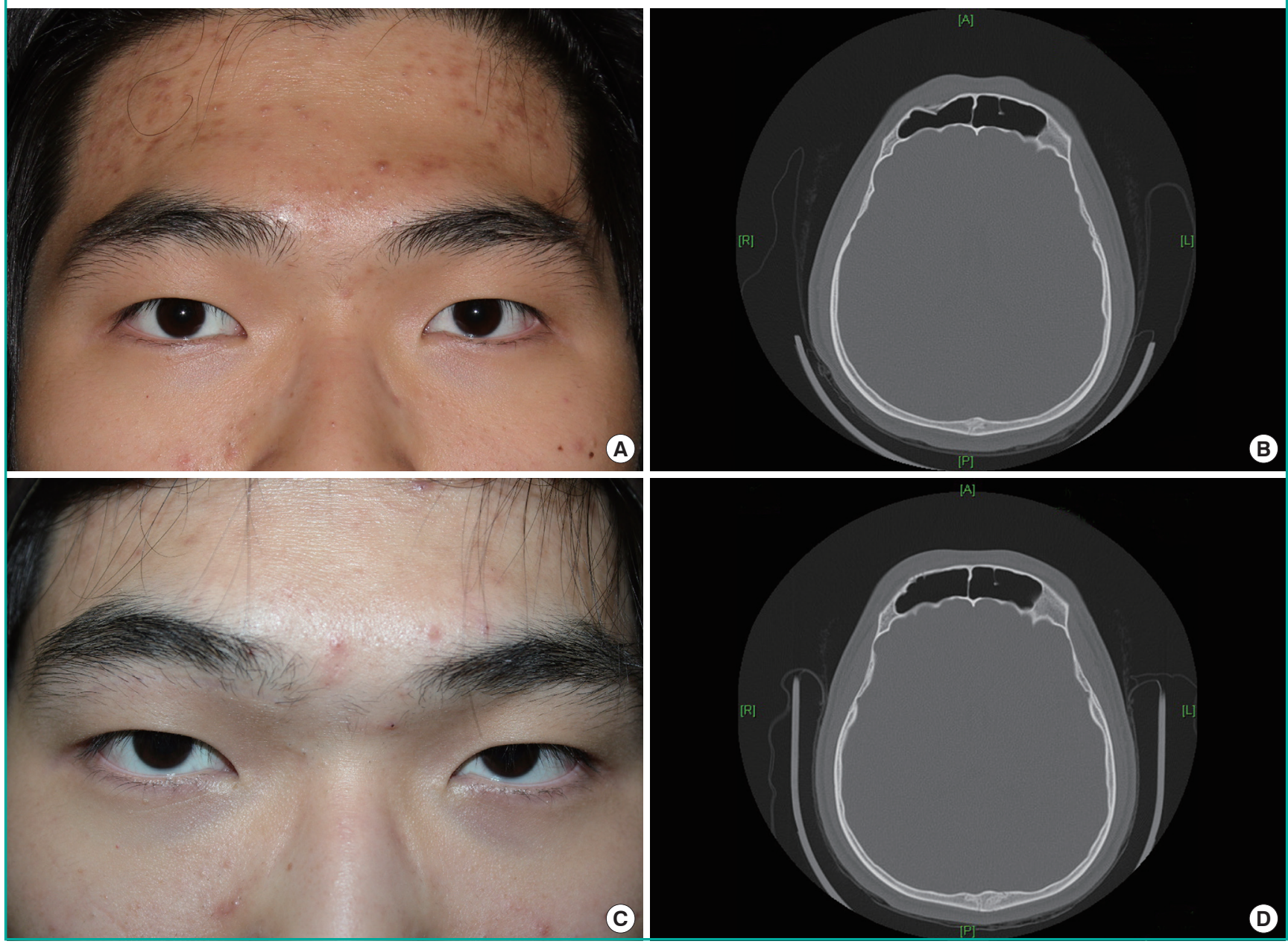

a three-hole absorbable plate and two absorbable screws. The patient did not show any transient numbness pre- or postoperatively. The follow-up CT scan showed a complete reduction of the fracture without any displacement. The postoperative photograph showed excellent healing of the scar (Fig. 4).

\section{DISCUSSION}

The period from the late 1800 s to the early 1900 s was the era of radical ablation procedures for frontal sinus fractures. The first operation for the fracture of the frontal sinus was described by Reidel [15] in 1989. Total exenteration of the frontal sinus left a disastrous cosmetic deformity. Over the decades, treatment methods have evolved to conservative procedures in order to avoid cosmetic defects $[16,17]$. Subsequent improvement in diagnostic imaging, such as CT scans and surgical technologies, in the past hundred years has led to a wide variety of philosophies, protocols, and procedures for the management of frontal bone fractures. Many agree that the management of frontal sinus fractures should be individualized on the basis of a clinical examination and diagnostic imaging in order to achieve the goal of providing an aesthetic outcome and a restoring function, and preventing complications. In recent years, early intervention with an emphasis on the cosmetic outcome with sinus preservation has gained general popularity [1,17-19]. It is reasonable that a simple isolated injury be treated through the use of a simple and effective method. Obliteration and cranialization procedures are performed only in more restrictive indications.

A bicoronal approach has been considered the standard approach in craniofacial surgery for many years. Patients who require obliteration or cranialization procedures require a bicoronal incision. Although a bicoronal incision offers adequate exposure, it requires a longer operative time and hospital stay. It has severe disadvantages, including a long scalp scar, alopecia, and temporal 
hollowing caused by extensive dissection $[4,5,10,11,20]$. The main problem for the isolated anterior wall fracture is the aesthetic deformity of the forehead, which seldom causes functional complications; therefore, if the surgical approach leaves more severe deformities, as described above, it should not be considered the treatment of choice. Many patients prefer a slight depression of the forehead rather than the long visible scar caused by a coronal incision. Open reduction of a frontal sinus fracture via a bicoronal incision has drawbacks for patients with simple forehead depressed fractures. Thus, in recent years, minimally invasive approaches to anterior table fractures have been used.

The endoscopic approach was first described by Graham and Spring [21] in 1996. The major bone fragments of an anterior table fracture with an intact posterior sinus wall were elevated without internal fixation. They reported that the patient had complete restoration of the cosmetic defect without postoperative complications. In 2003, Strong et al. [5] attempted endoscopic reduction and fixation in a cadaver study. They concluded that the degree of comminution dictated the success of the repair. When there were significant comminutions or marked fractures, rigid fixation could not be performed in a noninvasive manner. The endoscopic approach also has disadvantages, including a steep learning curve, narrow field of view, and lack of depth perception $[9,20]$.

Kim et al. [12] treated patients with anterior table frontal sinus fractures using a transcutaneous transfrontal approach through a small peri-eyebrow skin incision. However, rigid internal fixation is not possible if the skin incision is not extended to the fracture site, and in cases of a severely comminuted fracture, adequate reduction cannot be achieved since the transfer of a strong reduction force only by the insertion of a miniature periosteal elevator through the incision site is impossible.

Yoo et al. [13] performed a successful transcutaneous reduction of a frontal sinus fracture by using a bone tapper device with a 3-mm slit incision. This method involving the use of a tapper was considered easy to perform with relatively sufficient strength for handling a bony segment as compared to elevators. A 3-mm slit incision did not cause any problematic scars, but it was not adequate for the performance of the internal fixation of fractured segments.

Noury et al. [14], who treated anterior table frontal sinus fractures using frontal rhytid forehead incisions, concluded that this approach offered a good cosmetic result with the ability to perform internal fixation. However, their method is inappropriate for use in young patients with an invisible frontalis rhytid. In addition, there is still a risk of a long apparent scar across the forehead and paresthesia above the incision by an injury of the supratrochlear or supraorbital nerve.
Montovani et al. [22] treated patients with frontal sinus fractures by making a butterfly incision below the eyebrows, which provided adequate exposure not only for the performance of internal fixation but also for the performance of more complicated procedures such as obliteration and cranialization. It is difficult to hide the scar on the nasal dorsum, which connects the bilateral infrabrow incisions, and there is no need for bilateral infrabrow incisions in the case of the unilateral frontal sinus fracture. A butterfly incision may be considered too extensive for the treatment of an isolated anterior table fracture $[3,6,7]$.

The transcutaneous approach through a subbrow incision offers many advantages. An almost direct visualization of the fracture enables an accurate reduction of the anterior table of the frontal sinus. Rigid internal fixation, which was not possible by endoscopic and other minimal transcutaneous approaches, was performed in all cases. Minimization of the scar was achieved by camouflaging the scar at the lower margin of the eyebrow. This incision line is widely used in aesthetic blepharoplasty procedures and leaves an acceptable scar [23,24]. A subbrow incision is considered superior to the frontalis rhytid, butterfly, or bicoronal incisions in terms of the inconspicuous scar.

The main limitation of the transcutaneous approach through a subbrow incision is that rigid internal fixation could not be performed when the fracture was placed in the medial frontal sinus. Although the extent of periosteal dissection through the subbrow incision is from the origin of the temporalis muscle to beyond the midline of the forehead, internal fixation is not easy to perform when the fracture is placed around the midline. In such cases, a 5-7-mm slit incision for screw fixation was used for the achievement of rigid internal fixation. Development of numbness by the traction injury to the nerves during reduction and fixation is theoretically possible. However, in our experiences, complete identification and minimal handling of the nerves was possible because the subbrow incision provided a sufficient visual field. Therefore, there was no case of traction injury of the supratrochlear or supraorbital nerves.

Considering the surgical success of reduction and rigid fixation, patient satisfaction, and its aesthetic benefits, this transcutaneous approach through a subbrow incision is superior to other reduction techniques in the case of an anterior table frontal sinus fracture.

\section{REFERENCES}

1. Yavuzer R, Sari A, Kelly CP, et al. Management of frontal sinus fractures. Plast Reconstr Surg 2005; 115:79e-93e.

2. Metzinger SE, Guerra AB, Garcia RE. Frontal sinus fractures: management guidelines. Facial Plast Surg 2005;21: 
199-206.

3. Rohrich RJ, Hollier LH. Management of frontal sinus fractures. Changing concepts. Clin Plast Surg 1992;19:219-32.

4. Gossman DG, Archer SM, Arosarena O. Management of frontal sinus fractures: a review of 96 cases. Laryngoscope 2006;116:1357-62.

5. Strong EB, Buchalter GM, Moulthrop TH. Endoscopic repair of isolated anterior table frontal sinus fractures. Arch Facial Plast Surg 2003;5:514-21.

6. Helmy ES, Koh ML, Bays RA. Management of frontal sinus fractures. Review of the literature and clinical update. Oral Surg Oral Med Oral Pathol 1990;69:137-48.

7. Luce EA. Frontal sinus fractures: guidelines to management. Plast Reconstr Surg 1987;80:500-10.

8. Chen DJ, Chen CT, Chen YR, et al. Endoscopically assisted repair of frontal sinus fracture. J Trauma 2003;55:378-82.

9. Doonquah L, Brown P, Mullings W. Management of frontal sinus fractures. Oral Maxillofac Surg Clin North Am 2012; 24:265-74.

10. Steiger JD, Chiu AG, Francis DO, et al. Endoscopic-assisted reduction of anterior table frontal sinus fractures. Laryngoscope 2006;116:1936-9.

11. Strong EB, Kellman RM. Endoscopic repair of anterior table--frontal sinus fractures. Facial Plast Surg Clin North Am 2006;14:25-9.

12. Kim KS, Kim ES, Hwang JH, et al. Transcutaneous transfrontal approach through a small peri-eyebrow incision for the reduction of closed anterior table frontal sinus fractures. J Plast Reconstr Aesthet Surg 2010;63:763-8.

13. Yoo A, Eun SC, Baek RM. Transcutaneous reduction of frontal sinus fracture using bony tapper device. J Craniofac
Surg 2012;23:1835-7.

14. Noury M, Dunn RM, Lalikos JF, et al. Frontal sinus repair through a frontalis rhytid approach. Ann Plast Surg 2011; 66:457-9.

15. Reidel RA. Schenke: inaugural dissertation. Jena; 1898.

16. Rubin JS, Lund VJ, Salmon B. Frontoethmoidectomy in the treatment of mucoceles. A neglected operation. Arch Otolaryngol Head Neck Surg 1986;112:434-6.

17. Wolfe SA, Johnson P. Frontal sinus injuries: primary care and management of late complications. Plast Reconstr Surg 1988;82:781-91.

18. Koento T. Current advances in sinus preservation for the management of frontal sinus fractures. Curr Opin Otolaryngol Head Neck Surg 2012;20:274-9.

19. Bell RB, Dierks EJ, Brar P, et al. A protocol for the management of frontal sinus fractures emphasizing sinus preservation. J Oral Maxillofac Surg 2007;65:825-39.

20. Strong EB. Endoscopic repair of anterior table frontal sinus fractures. Facial Plast Surg 2009;25:43-8.

21. Graham HD 3rd, Spring P. Endoscopic repair of frontal sinus fracture: case report. J Craniomaxillofac Trauma 1996; 2:52-5.

22. Montovani JC, Nogueira EA, Ferreira FD, et al. Surgery of frontal sinus fractures: epidemiologic study and evaluation of techniques. Braz J Otorhinolaryngol 2006;72:204-9.

23. Ichinose A, Sugimoto T, Sugimoto I, et al. Extended infrabrow excision blepharoplasty for dermatochalasis in asians. Arch Facial Plast Surg 2011;13:327-31.

24. Kim YS, Roh TS, Yoo WM, et al. Infrabrow excision blepharoplasty: applications and outcomes in upper blepharoplasty in Asian women. Plast Reconstr Surg 2008;122:1199-205. 